\title{
Flux Qubits and Readout Device with Two Independent Flux Lines
}

\author{
B.L.T. Plourde, T.L. Robertson, P.A. Reichardt, T. Hime, S. Linzen, C.-E. Wu, and John Clarke \\ Department of Physics, University of California, Berkeley, CA 94720-7300
}

(Dated: November 14, 2018)

\begin{abstract}
We report measurements on two superconducting flux qubits coupled to a readout Superconducting QUantum Interference Device (SQUID). Two on-chip flux bias lines allow independent flux control of any two of the three elements, as illustrated by a two-dimensional qubit flux map. The application of microwaves yields a frequency-flux dispersion curve for 1- and 2-photon driving of the single-qubit excited state, and coherent manipulation of the single-qubit state results in Rabi oscillations and Ramsey fringes. This architecture should be scalable to many qubits and SQUIDs on a single chip.
\end{abstract}

PACS numbers: 03.67.Lx, 85.25.Cp, 85.25.Dq

Superconducting quantum bits (qubits) based on charge [1, 2], magnetic flux 3, 4], and phase difference across a Josephson junction [5, 6] are attractive candidates for the basis of a quantum computer because of their inherent scalability using established thin-film fabrication techniques. Advantages of the flux qubit include its immunity to the ubiquitous charge noise in the substrate and that it can be configured with no direct electrical connections. One type of flux qubit consists of a superconducting loop interrupted by three Josephson junctions with critical currents $I_{0}, I_{0}$, and $\alpha I_{0}(\alpha<1)[7]$. When the applied flux bias $\Phi_{Q}$ is at a degeneracy point $(n+1 / 2) \Phi_{0}\left(n\right.$ is an integer such that $\left|\Phi_{Q}-n \Phi_{0}\right| \leq \Phi_{0} / 2$; $\Phi_{0} \equiv h / 2 e$ is the flux quantum), a screening supercurrent $J_{Q}$ can flow in either direction around the loop. The ground and first excited states of the qubit correspond to symmetric and antisymmetric superpositions of the two current states and are separated by an energy $\Delta$. Here, $\Delta / h$ is the tunnel frequency between the current states, typically a few GHz. When $\Phi_{Q}$ is away from a degeneracy point, the energy difference between the two superposed states is $\nu=\left(\Delta^{2}+\epsilon^{2}\right)^{1 / 2}$, where $\epsilon=2 J_{Q}\left[\Phi_{Q}-(n+1 / 2) \Phi_{0}\right]$. The state of the qubit is measured by coupling the screening flux generated by $J_{Q}$ to a hysteretic dc superconducting quantum interference device (SQUID). This flux determines the bias current at which the SQUID switches out of the zero-voltage state.

In addition to the development of scalable interqubit couplings [8], a prerequisite for scaling to a system of many qubits is that the attendant readout, filtering, and bias circuitry also scale. A particular challenge is that the flux bias must be settable for each element individually. This mandates the use of on-chip flux-bias lines in an arrangement that enables one to apply a combination of currents to address any given qubit or SQUID while maintaining all other flux biases at constant values. Furthermore, the bias currents required to change the flux over (say) $\pm 1 \Phi_{0}$ should not be so large that it becomes impractical to deliver them to a chip cooled to millikelvin temperatures. This requirement establishes minimum self-inductances of the qubit and readout SQUID that are substantially larger than values used previously in 3-junction qubits, which have relied on external coils to generate large magnetic fields $[4,9,10]$. At the same time, the mutual inductance between the on-chip flux lines and the qubit must be sufficiently small for the noise generated by the circuitry supplying the flux bias current not to be the limiting source of decoherence 11].

In this Letter, we report measurements on two qubits and a readout SQUID that meet these criteria. We illustrate the orthogonalization of the applied fluxes by means of a two-dimensional flux map. We report spectroscopy on one of the two qubits that matches the expected dispersion for a flux qubit. In addition, we observe spurious resonances, some of which may be related to defects in the tunnel barriers of the qubit. We perform coherent manipulation of the single-qubit state resulting in Rabi oscillations and Ramsey fringes. In prior measurements of coherent oscillations [9, 10], the readout SQUID was connected directly to the flux qubit and thus detected a combination of flux and phase changes. In contrast, our SQUID is electrically isolated from the qubit and detects only flux changes.

Figure 1 shows the device layout. The readout SQUID has a calculated inductance $L_{S}=358 \mathrm{pH}$, and each of the two qubits it encloses has a calculated inductance $L_{Q}=143 \mathrm{pH}$. The calculated mutual inductance between each qubit and the SQUID is $61 \mathrm{pH}$. One pair of seriesconnected flux bias lines is arranged near the top of the SQUID and a second near the bottom; thus, flux in qubit 1 (2) is supplied predominantly by the lower (upper) flux lines. The mutual inductance between each qubit and its associated flux lines was designed to be $4-5 \mathrm{pH}$, enabling us to apply $\sim 2 \Phi_{0}$ with a current of $1 \mathrm{~mA}$. This criterion dictated the relatively large qubit self-inductances compared with those in previous experiments [4, 9, 12, 13.

We fabricated the device on an oxidized Si substrate using electron-beam lithography and double-angle evaporation to form the $\mathrm{Al}-\mathrm{AlOx}-\mathrm{Al}$ tunnel junctions. The $\mathrm{Al}$ lines for the qubit and SQUID loops were $1 \mu \mathrm{m}$ wide, and those for the flux bias $10 \mu \mathrm{m}$ wide. Each SQUID junction was $175 \times 200 \mathrm{~nm}^{2}$ with a critical current of 


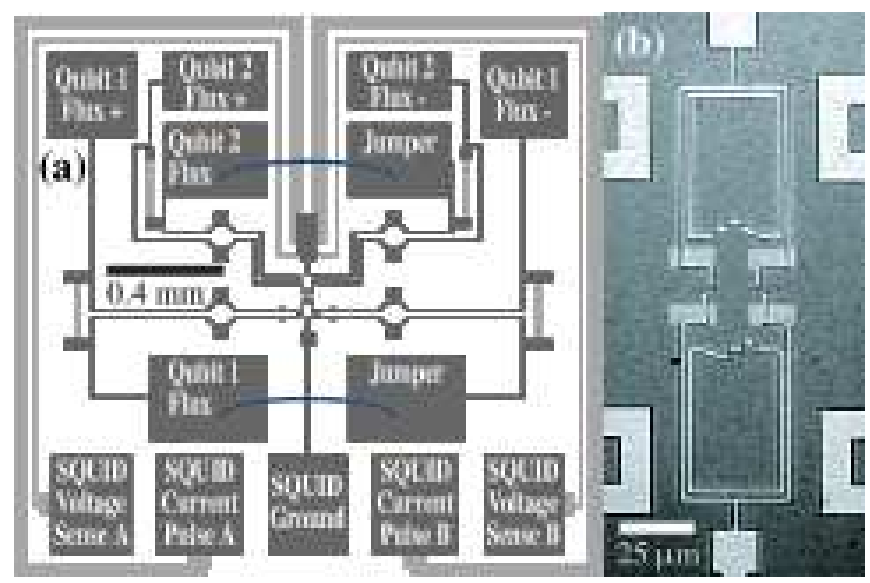

FIG. 1: (a) Chip layout. Dark gray represents Al traces, light gray $\mathrm{AuCu}$ traces. Pads near upper edge of chip provide two independent flux lines; wirebonded Al jumpers couple left and right halves. Pads near lower edge of chip supply current pulses to the readout SQUID and sense any resulting voltage. (b) Photograph of center region of completed device. Segments of flux lines are visible to left and right of SQUID, which surrounds the two qubits.

$220 \mathrm{nA}$. For qubits 1 and 2, the larger junctions had areas of $250 \times 250 \mathrm{~nm}^{2}$ and $180 \times 200 \mathrm{~nm}^{2}$, approximate critical currents $I_{0}$ (scaled from SQUID junction areas) of $390 \mathrm{nA}$ and $230 \mathrm{nA}$, and $\alpha$-values (based on junction areas) of 0.49 and 0.68 , respectively. The different junction parameters were chosen to increase the probability of obtaining one viable qubit; in fact, qubit 2 displayed good characteristics. A $42 \mathrm{~nm}$-thick AuCu film deposited and patterned prior to the $\mathrm{Al}$ deposition provided quasiparticle traps near the junctions [14], $100 \Omega$ shunts on each of the four flux lines, and $500 \Omega$ and $1275 \Omega$ series resistors on each end of the pulse lines and sense lines, respectively.

To eliminate external magnetic field fluctuations we enclosed the chip in a $6 \times 16 \times 22 \mathrm{~mm}^{3}$ cavity machined into a copper block and plated with $\mathrm{Pb}$. This superconducting cavity stabilizes the field, enabling us to acquire data for periods up to 48 hours. A 1 mm-diameter superconducting loop $\sim 3 \mathrm{~mm}$ above the chip supplied microwave flux. The sample holder was attached to the mixing chamber of a dilution refrigerator at $50 \mathrm{mK}$. All electrical leads to the experiment were heavily filtered at several different temperatures with a combination of lumped circuit and copper powder low-pass filters [15]. Measurements of the state of a qubit as a function of flux, microwave power and frequency were made by pulsing the current in the SQUID and detecting whether or not it switched out of the zero-voltage state by means of a low-noise, room-temperature amplifier. The flux bias currents were supplied by highly stable potentiometers, controlled by a computer over a fiber-optic link [16].

There are three applied fluxes that determine the state of the system: the SQUID flux $\Phi_{S}$, and the qubit fluxes $\Phi_{Q 1}$ and $\Phi_{Q 2}$. Given the two flux lines, we can set any two fluxes arbitrarily; the third is fully constrained. This can be expressed succinctly by the matrix equation

$$
\left[\begin{array}{lll}
\Phi_{S} & \Phi_{Q 1} & \Phi_{Q 2}
\end{array}\right]=\left[\begin{array}{ccc}
M_{F 1 S} & M_{F 2 S} & \Phi_{S}^{0} \\
M_{F 1 Q 1} & M_{F 2 Q 1} & \Phi_{Q 1}^{0} \\
M_{F 1 Q 2} & M_{F 2 Q 2} & \Phi_{Q 2}^{0}
\end{array}\right]\left[\begin{array}{c}
I_{1} \\
I_{2} \\
1
\end{array}\right]
$$

where the $M_{i j}$ are mutual inductances between the various flux lines and loops, the $\Phi_{j}^{0}$ account for static background fields, and the $I_{i}$ are currents in the flux lines.

We turn now to our experimental results. Figure 2(a) shows the switching probability of the SQUID versus the amplitude of the current pulses applied to it for two different values of $\Phi_{Q 2}$ at constant $\Phi_{S}$. Because we hold $\Phi_{S}$ constant, we can measure the displacement of the two curves for constant sensitivity in the SQUID. The measurement fidelity, which is the difference between the switching probabilities, has a maximum of about $60 \%$. Figure 2(b) shows $I_{s}^{50 \%}$ vs. $\Phi_{S} ; I_{s}^{50 \%}$ is the pulse amplitude for which the switching probability is $50 \%$. The effects of the changing flux in the qubits are small on this scale. Figure 2(c) shows $I_{s}^{50 \%}$ vs. $\Phi_{Q 1}$ for constant $\Phi_{S}$; as $\Phi_{Q 1}$ is varied, a flux of approximately the same magnitude and opposite sign is applied to qubit 2. Thus, as $\Phi_{Q 1}$ is increased at constant $\Phi_{S}, I_{s}^{50 \%}$ abruptly increases when qubit 1 flips state and decreases when qubit 2 flips state.

To determine the parameters in Eq. (11), we sampled $I_{s}^{50 \%}$ at $\sim 20,000$ different settings of $I_{1}$ and $I_{2}$. We fit these data to a parametric model of the response of the SQUID $I_{s}^{f i t}(\Phi)$ to the total SQUID flux $\Phi_{T}$. To describe the SQUID modulation, we use the ad hoc expression

$$
I_{s}^{f i t}(\Phi)=\sum_{i=1}^{15} a_{i} \cos 2 \pi i \frac{\Phi}{\Phi_{0}}+\sum_{i=2}^{15} b_{i} \sin 2 \pi i \frac{\Phi}{\Phi_{0}}+d,
$$

where the $a_{i}, b_{i}$, and $d$ are fit parameters. The total flux coupled to the SQUID is well approximated by

$$
\Phi_{T}=\Phi_{S}+\Delta \Phi_{Q 1} j_{Q 1}\left(\Phi_{Q 1}\right)+\Delta \Phi_{Q 2} j_{Q 2}\left(\Phi_{Q 2}\right)
$$

which neglects the self-screening of the SQUID. Here, the $\Delta \Phi_{Q i}=J_{Q i} M_{Q i S}$ are the amplitudes of qubit screening flux changes near the degeneracy point, referred to the SQUID, and the qubit circulating currents are described by dimensionless periodic functions of unit amplitude

$$
j_{Q i}(\Phi)=\left\{\sin \left[2 \pi g_{Q i}\left(\Phi-n \Phi_{0}\right) / \Phi_{0}\right]\right\} / \sin \pi g_{Q i},
$$

with fitting parameters $g_{Q i}$. The $j_{Q i}(\Phi)$ are discontinuous when $n$ increments.

Fitting the $I_{s}^{50 \%}\left(I_{1}, I_{2}\right)$ data, we obtain the following parameters for $I_{s}^{f i t}\left(\Phi_{T}\right): M_{F 1 Q 1}=3.96 \mathrm{pH}, M_{F 2 Q 1}=$ $-0.77 \mathrm{pH}, M_{F 1 Q 2}=1.37 \mathrm{pH}, M_{F 2 Q 2}=-3.31 \mathrm{pH}$, $M_{F 1 S}=8.30 \mathrm{pH}, M_{F 2 S}=-6.30 \mathrm{pH}, \Delta \Phi_{Q 1}=4.39$ 


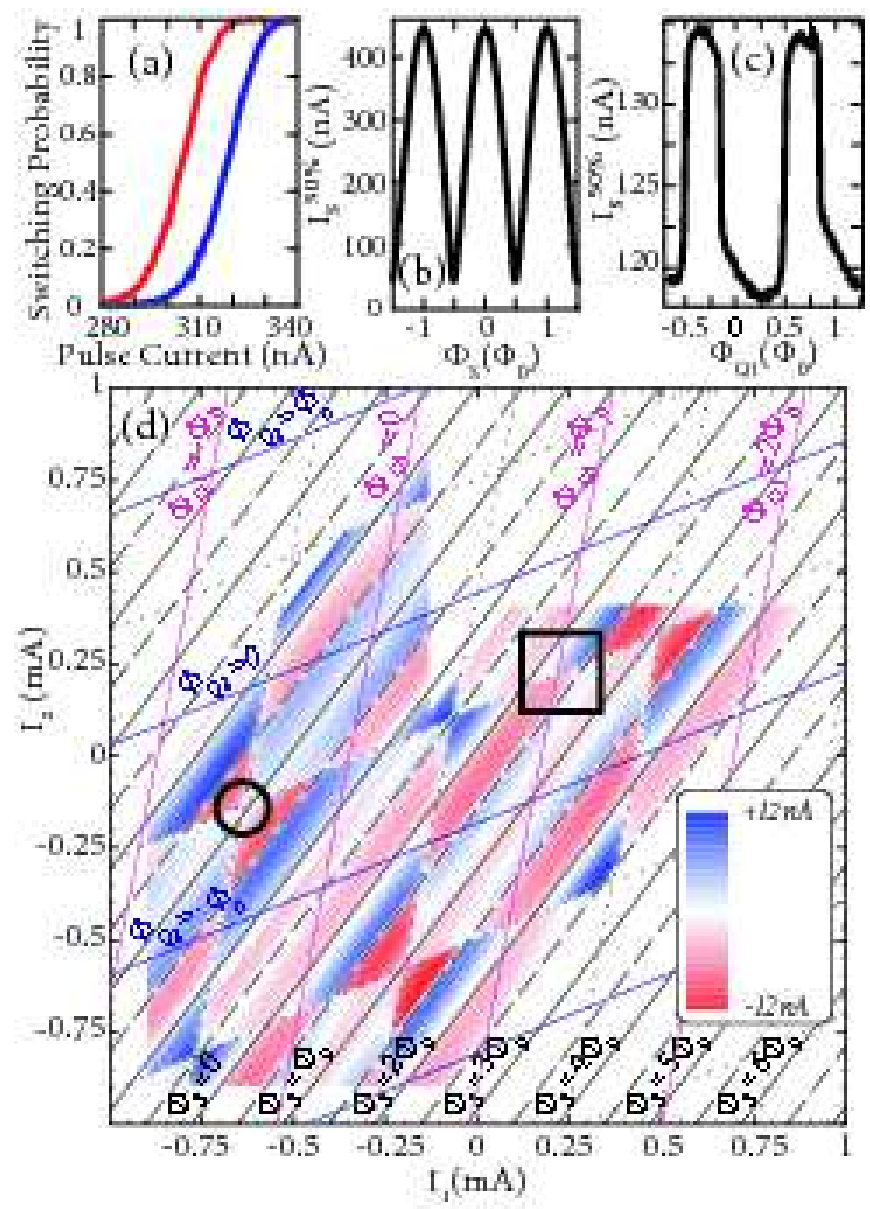

FIG. 2: (a) SQUID switching probability vs. amplitude of bias current pulse near qubit 2 transition. The two curves represent the states corresponding to $\Phi_{Q 2}=0.48 \Phi_{0}$ (red) and $\Phi_{Q 2}=0.52 \Phi_{0}$ (blue); $\Phi_{S}$ is held constant. Each curve contains 100 points averaged 8,000 times. (b) $I_{s}^{50 \%}$ vs. $\Phi_{S}$. Each period of oscillation contains $\sim 5,000$ flux values, and each switching current is averaged 8,000 times. (c) Dependence of $I_{s}^{50 \%}$ on $\Phi_{Q 1}$ for constant $\Phi_{S}$. (d) Qubit flux map.

$\mathrm{m} \Phi_{0}, \Delta \Phi_{Q 2}=4.04 \mathrm{~m} \Phi_{0}, g_{Q 1}=0.64, g_{Q 2}=0.51$. This fit captures all the essential features of the data, having a root-mean-square residual of $1.8 \mathrm{nA}$, that is, less than $0.5 \%$ of the maximum value of $I_{s}^{50 \%}$.

In Fig. 2(d), we plot $I_{s}^{50 \%}-I_{s}^{f i t}\left(\Phi_{S}\right)$ versus the currents $I_{1}$ and $I_{2}$; lines of constant $\Phi_{S}, \Phi_{Q 1}$, and $\Phi_{Q 2}$ are indicated. This qubit flux map displays only the flux contributions of the two qubits. For example, inside the square, where $\Phi_{Q 1} \approx \Phi_{0}$ and $\Phi_{Q 2} \approx-\Phi_{0} / 2$, $I_{s}^{50 \%}$ exhibits an abrupt step across the $\Phi_{Q 2}$ line, as $J_{Q 2}$ changes direction discontinuously. However, there is no step across the $\Phi_{Q 1}$ line, as $J_{Q 1}$ is continuous. Furthermore, one can scan the switching current along a line where $\Phi_{S}$ is held constant at an arbitrary value, to observe the effects of flux only on the qubits. Finally, special points where the two qubits are each at a degeneracy

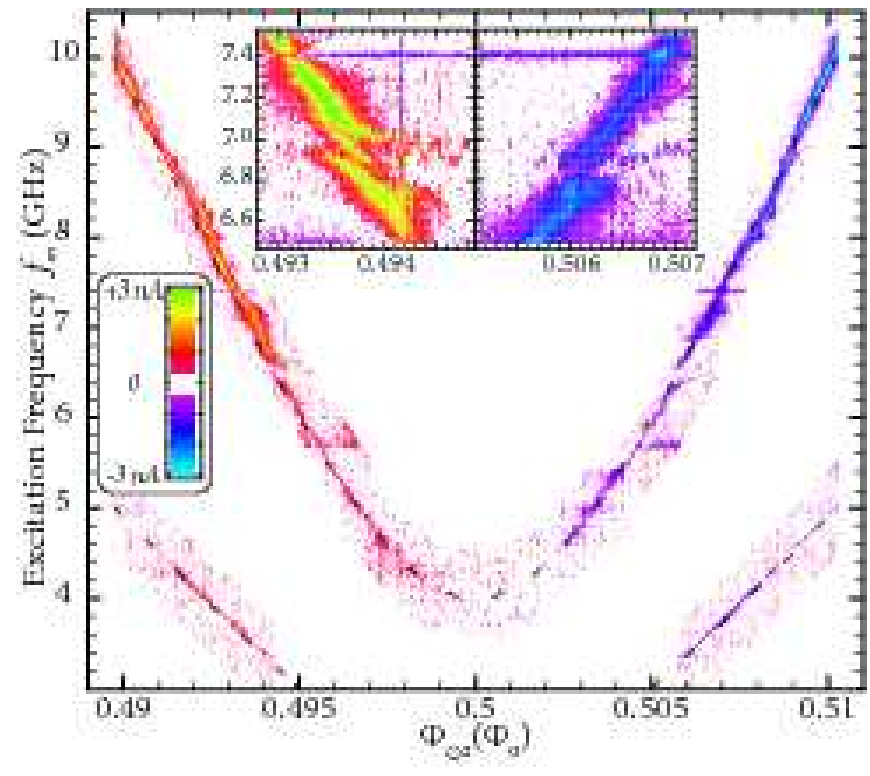

FIG. 3: Spectroscopy of qubit 2. Enhancement and suppression of $I_{s}^{50 \%}$ is shown as a function of $\Phi_{Q 2}$ and $f_{m}$ relative to measurements in the absence of microwaves. Dashed lines indicate fit to hyperbolic dispersion for 1- and 2-photon qubit excitations. The 2-photon fit is one-half the frequency of the 1-photon fit. Inset containing $\sim 23,000$ points is at higher resolution.

point are indicated by the circle.

The remaining discussion of the experiments is concerned with the effects of an applied microwave flux on qubit 2. Photons of energy $h f_{m}=\nu$ drive transitions between the two qubit states, producing peaks and dips on the qubit transitions. By measuring $I_{s}^{50 \%}$ as a function of $\Phi_{Q 2}$ and $f_{m}$, we determined the dispersion relation shown in Fig. 3. This measurement contains a total of $\sim 75,000$ points and took 48 hours to acquire, thus demonstrating the excellent flux stability in our system. The dispersion is well described by the hyperbolic relation $\nu=\left(\epsilon^{2}+\Delta^{2}\right)^{1 / 2}$, with $\Delta / h=(3.99 \pm 0.05) \mathrm{GHz}$ and $(1 / h) d \epsilon / d \Phi_{Q 2}=(896 \pm 5) \mathrm{MHz} / \mathrm{m} \Phi_{0}$. Part of the spectrum corresponding to 2-photon transitions is also shown.

Figure 3 shows two types of deviation from ideal behavior, more evident in the inset which was measured 4 weeks before the full spectrum. First, there are sharp suppressions of the critical current which are independent of $\Phi_{Q 2}$ and hence occur at particular constant values of $f_{m}$, for example, at $7.4 \mathrm{GHz}$, that we believe arise from electromagnetic modes which couple to the qubit. Second, we see disruptions of the dispersion curve that are suggestive of coupling between the qubit and other two-state systems. One instance of this second anomaly, originally centered near $7 \mathrm{GHz}$ in the inset and shifted to $6.5 \mathrm{GHz}$ in the full spectrum, is remarkably similar to those reported for phase qubits, and may be of the same 


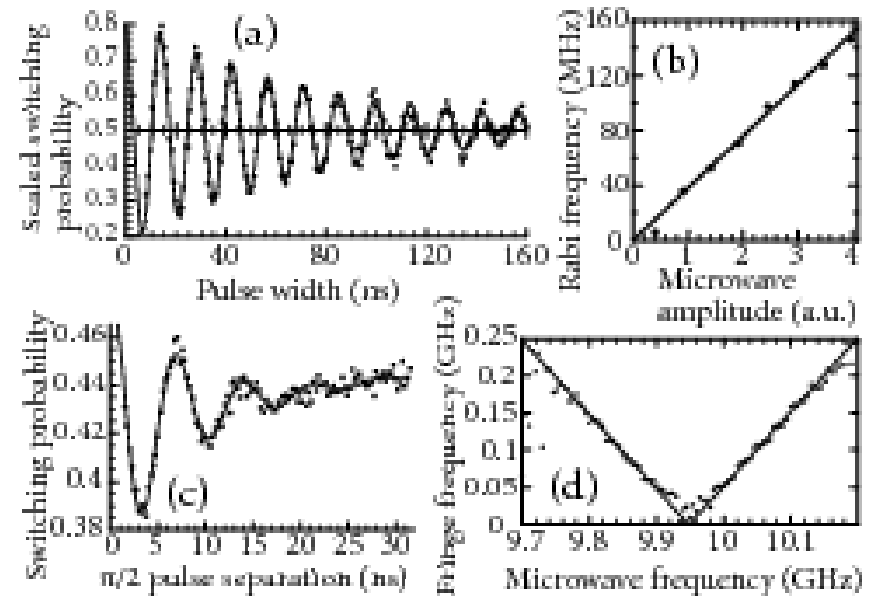

FIG. 4: Coherent manipulation of qubit state. (a) Rabi oscillations, scaled to measured SQUID fidelity, as a function of width of $10.0 \mathrm{GHz}$ microwave pulses. (b) Rabi frequency vs. $10.0 \mathrm{GHz}$ pulse amplitude; line is least squares fit to the data. (c) Ramsey fringes for qubit splitting of $9.95 \mathrm{GHz}$, microwave frequency of $10.095 \mathrm{GHz}$. (d) Ramsey fringe frequency vs. microwave frequency. Lines with slopes \pm 1 are fits to data.

origin [17.

We performed coherent manipulation of the qubit state by varying the duration of a resonant microwave pulse for fixed frequency and amplitude. Upon applying fixedamplitude bias current pulses, we observed Rabi oscillations in the switching probability of the SQUID as a function of the microwave pulse width at approximately 100 frequencies ranging from 4.37 to $13.86 \mathrm{GHz}$. Generally speaking, the amplitude of the oscillations is higher and their decay time is longer for qubit flux bias points away from the spurious splittings shown in the spectroscopy. An example is shown in Fig. 4(a), where each of the 195 points was averaged 20,000 times. We reference the amplitude of these oscillations to the qubit by scaling to the SQUID measurement fidelity. We observed Rabi oscillations with the longest decay time when we operated at a bias current of $296 \mathrm{nA}$, where the fidelity was $14.4 \%$ for this particular value of $\Phi_{S}$ and the SQUID switched infrequently [Fig. 2(a)]. We believe that this improvement is related to noise currents in the SQUID loop which couple to the qubit through $M_{Q 2 S}$ and are produced by the quasiparticles generated in the SQUID loop during each switching event: a lower SQUID switching probability results in fewer quasiparticles averaged over time [18]. Switching probability data in Fig. 4(a), scaled to the measurement fidelity of the SQUID, fit well to a damped sinusoid with a visibility of $63 \%$ and a decay time $\tau_{R a b i}=78 \mathrm{~ns}$. Fits to similar measurements for different microwave pulse amplitudes show that the Rabi frequency scales linearly with the microwave amplitude [Fig. 4(b)], as expected for coherent driving [19].

We measured the decay of a resonance peak and ob- tained the relaxation time $\tau_{R}=281 \mathrm{~ns}$ at $10.0 \mathrm{GHz}$ from an exponential fit. We measured the dephasing time $\tau_{\phi}$ from Ramsey fringes [20]. We first applied a $\pi / 2$ pulse to tip the qubit state vector into the equatorial plane, where it dephased. To calibrate the $\pi / 2$ pulses, we chose a microwave pulse width based on a Rabi oscillation measurement made at the same operating point. After a variable time delay $\tau$, we applied a second $\pi / 2$ pulse followed by a measurement of the SQUID switching probability for a fixed bias current pulse amplitude. When we chose a microwave frequency off resonance we observed damped oscillations of the SQUID switching probability [Fig. 4(c)] with a fit decay time of the oscillations $\tau_{\phi}=6.6 \mathrm{~ns}$. Figure 4(d) shows the Ramsey fringe frequency for 100 values of the microwave frequency, together with the fit to two lines with slopes of magnitude unity, as expected for coherently driven Ramsey fringes.

In conclusion, we have demonstrated on-chip flux bias lines which allow us to vary the flux applied to two of the three devices independently. The mutual inductance between the flux lines and the qubits is weak enough that the characteristic impedance of the flux lines (say $50 \Omega$ ) should not limit our coherence times [1]. Although in this Letter we have concentrated on the quantum coherent properties of a single flux qubit, we note that the two flux qubits in this design in principle could be coupled controllably using the circulating currents in the dc SQUID [8]. This system of qubits, SQUID and flux lines should be readily scalable.

We thank M.H. Devoret, D. Esteve, C.J.P.M. Harmans, J.M. Martinis, R. McDermott, J.E. Mooij, R.J. Schoelkopf, D. Vion, and F.K. Wilhelm for helpful discussions. This work was supported by the Air Force Office of Scientific Research under Grant F49-620-02-1-0295, the Army Research Office under Grant DAAD-19-02-1-0187, the National Science Foundation under Grant EIA-0205641, and the Advanced Research and Development Activity.

[1] Y. Nakamura, Y. A. Pashkin, and J. S. Tsai, Nature 398, 786 (1999).

[2] D. Vion et al., Science 296, 286 (2002).

[3] J. Friedman et al., Nature 46, 43 (2000).

[4] C. H. van der Wal et al., Science 290, 773 (2000).

[5] J. M. Martinis et al., Phys. Rev. Lett. 89, 117901 (2002).

[6] R. C. Ramos et al., IEEE Trans. Appl. Supercon. 11, 998 (2001).

[7] J. E. Mooij et al., Science 285, 1036 (1999).

[8] B. L. T. Plourde et al., Phys. Rev. B 70, 140501(R) (2004).

[9] I. Chiorescu et al., Science 299, 1869 (2003).

[10] I. Chiorescu et al., Nature 431, 159 (2004).

[11] Y. Makhlin, G. Schön, and A. Shnirman, Rev. Mod. Phys. 73, 357 (2001).

[12] S. Saito et al., Phys. Rev. Lett. 93, 037001 (2004). 
[13] E. Il'ichev et al., Phys. Rev. Lett. 91, 097906 (2003).

[14] K. Lang et al., IEEE Trans. Appl. Supercond. 13, 989 (2003).

[15] J. M. Martinis, M. H. Devoret, and J. Clarke, Phys. Rev. B 35, 4682 (1987).

[16] S. Linzen et al., Rev. Sci. Inst. 75, 2541 (2004).

[17] R. W. Simmonds et al., Phys. Rev. Lett. 93, 077003
(2004).

[18] P. A. Reichardt et al., Bull. Am. Phys. Soc. 49, A37.004 (2004).

[19] I. I. Rabi, Phys. Rev. 51, 652 (1937).

[20] N. F. Ramsey, Phys. Rev. 78, 695 (1950). 